\title{
On Bounded Solutions of a Classical Yang-Mills Equation
}

\section{Michael Renardy}

Institut für theoretische Physik, Universität Stuttgart, Pfaffenwaldring 57, D-7000 Stuttgart 80, Federal Republic of Germany

Abstract. We discuss bounded solutions of the equation

$$
r^{2}\left(\frac{\partial^{2} u}{\partial r^{2}}+\frac{\partial^{2} u}{\partial t^{2}}\right)=u^{3}-u
$$

in the halfspace $r>0$. All solutions depending only on $t / r$ are characterized topologically. Then we prove the existence of infinite dimensional manifolds of $t$-periodic as well as nonperiodic solutions which are small in a suitable norm.

\section{Introduction}

It was shown recently by Glimm and Jaffe [1] that multimeron solutions to the classical SU(2) Yang-Mills field equations in Euclidean space are characterized by the following singular elliptic boundary value problem:

$$
\begin{gathered}
r^{2}\left(\frac{\partial^{2} u}{\partial r^{2}}+\frac{\partial^{2} u}{\partial t^{2}}\right)=u^{3}-u \quad t \in R, \quad r>0 \\
\lim _{r, t \rightarrow \infty} u(r, t)=1, \quad u(0, t)=(-1)^{i} \quad \text { for } \quad t_{i}<t<t_{i+1}(i=0,1, \ldots, 2 n),
\end{gathered}
$$

where $-\infty=t_{0}<t_{1}<\ldots<t_{2 n-1}<t_{2 n}<t_{2 n+1}=\infty$.

Jonsson et al. proved in [2] that this boundary value problem has at least one solution for every choice of the $t_{i}$. In this paper we investigate some kinds of bounded solutions to $(0.1)$, which satisfy different boundary conditions.

We first prove (Sect. 1) that a bounded solution of $(0.1)$ which has a continuous extension to the $t$-axis except for a countable number of points must satisfy $|u| \leqq 1$ in the whole half-plane and cannot be positive everywhere, unless it is constant.

The special solutions which we discuss then are of two different types. In Sect. 2 we are concerned with solutions depending only the independent variable $\frac{t}{r}$, for which $(0.1)$ is reduced to an ordinary differential equation; in Sects. 3 and 4 we discuss solutions which are "small" in a suitable norm. 
Solutions depending only on $r$ or only on $r /\left(r^{2}+t^{2}\right)$ have been considered by Protogenov [5]. A second class of solutions for which (0.1) is reduced to an ODE are those depending only on $t / r=: \tau$. We prove in Sect. 2 that there exists a two dimensional continuum of such solutions satisfying $\lim _{\tau \rightarrow \pm \infty} u(\tau)=0$. By a limit procedure we find that there exists one (unique) solution assuming the boundary values $\lim _{\tau \rightarrow-\infty} u(\tau)=-1$ and $\lim _{\tau \rightarrow \infty} u(\tau)=1$. In addition we find a one dimensional continuum of solutions approaching \pm 1 only on one side and 0 on the other side.

Small solutions are discussed in Sect. 3 for the case of solutions that are periodic in $t$ with a given period and in Sect. 4 for a certain class of nonperiodic solutions. In both cases we find a one-to-one correspondence between small solutions and the nullspace of the linearization. This proves the existence of an infinite-dimensional manifold of bounded solutions. All these solutions approach 0 as $r \rightarrow 0$.

\section{A Priori Estimates for Bounded Solutions}

Theorem 1.1. Let $u$ be a bounded $C^{2}$-solution of $(0.1)$ in $r>0$ which can be continuously extended to the axis $r=0$ except at a countable number of points. Then $|u| \leqq 1$ in the whole halfplane $r>0$.

Remark. The condition that $u$ is $C^{2}$ is not really a restriction. As proved in [2], Theorem 3.1, every weak solution to $(0.1)$ which is in $L^{\infty}$ is real analytic in $r>0$.

Proof. (i) Let $\left(0, t_{0}\right)$ be a point on the $t$-axis where $u$ has a continuous limit. We are going to prove that $u\left(0, t_{0}\right)$ must take one of the values $0, \pm 1$. According to Green's formula we have

$$
\begin{aligned}
u(r, t) & =-\int_{\partial G} u\left(r_{1}, t_{1}\right) \frac{\partial \Gamma\left(r, t, r_{1}, t_{1}\right)}{\partial v} d \sigma-\int_{G} \Delta u\left(r_{1}, t_{1}\right) \Gamma\left(r, t, r_{1}, t_{1}\right) d r_{1} d t_{1} \\
& =-\int_{\partial G} \ldots-\int_{G} r_{1}^{-2}\left(u^{3}\left(r_{1}, t_{1}\right)-u\left(r_{1}, t_{1}\right)\right) \Gamma\left(r, t, r_{1}, t_{1}\right) d r_{1} d t_{1} .
\end{aligned}
$$

Here $\Gamma$ is Green's function for the bounded domain $G \subset R_{+}^{2}$.

Assume $u\left(0, t_{0}\right) \neq 0, \pm 1$ and let $U$ be a neighbourhood of $\left(0, t_{0}\right)$, in which $u$ never takes the values $0, \pm 1$. Let now $G$ be a square as shown in the next diagram.

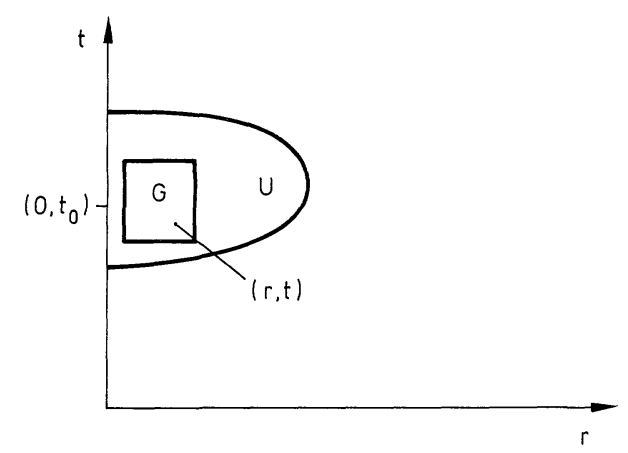

Fig. 1.1 
The second integral in the formula above diverges as the left boundary of $G$ is shifted to the $t$-axis (the integrand contains a factor $r_{1}^{-2}$, and only one $r_{1}$ is compensated by the Green's function, since the normal derivative of $\Gamma$ on a smooth portion of the boundary does not vanish [6]). This contradicts the boundedness of $u$.

(ii) The following argument modifies an idea of Glimm and Jaffe [1]. Let $F$ be a $C^{\infty}$-function $R \rightarrow R$ with the following properties: $F(u)=u$ for $u \leqq 1$ and $F(u)<u$ for $u>1, F^{\prime} \leqq 1, F^{\prime \prime} \leqq 0$. Then

$$
\Delta F(u)=F^{\prime} \Delta u+F^{\prime \prime}(\nabla u)^{2} \leqq F^{\prime} \Delta u \leqq \Delta u .
$$

Hence $u-F(u)$ is non-negative, subharmonic, bounded, and is equal to 0 on the $t$-axis except at a countable number of points. By inversion with respect to a circle we can map the half-plane onto the interior of a circle. From $u-F(u)$ we obtain then a function, which is non-negative, subharmonic and bounded in the interior of the cricle and vanishes on the boundary with at most countably many exceptional points. From [3, p. 204] we conclude that $u-F(u)$ is non-positive. This proves $u \leqq 1$, and replacing $u$ by $-u$ we find $u \geqq-1$.

Next we prove the non-existence of non-trivial positive solutions.

Theorem 1.2. Let $u \geqq 0$ be a bounded solution to (0.1) which can be continuously extended to the $t$-axis with at most countably many exceptional points. Then $u=1$ or $u=0$.

Proof. Since $u \geqq 0$ we have $\Delta u \leqq 0$, and if the boundary value is 1 everywhere on the $t$-axis, then $u=1$. So we may assume there exists a point $\left(0, t_{0}\right)$ such that $\lim u(r, t)=0$. Let $\zeta(t)$ be a cut-off function supported by a sufficiently small $(r, t) \rightarrow\left(0, t_{0}\right)$ interval containing $t_{0}$.

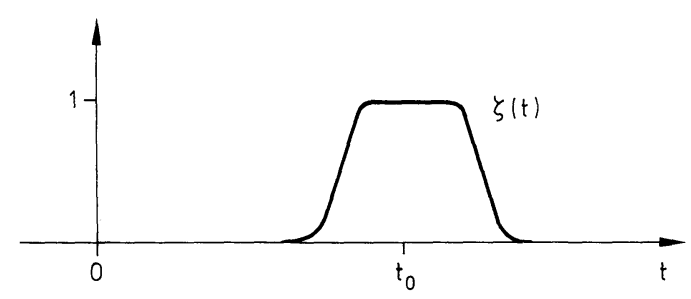

Fig. 1.2

$$
\begin{aligned}
& \text { We define } v(r)=\int_{-\infty}^{\infty} \zeta(t) u(r, t) d t \text {. Then Eq. (0.1) implies } \\
& \qquad \begin{aligned}
r^{2} \frac{d^{2} v}{d r^{2}} & =-v+\int_{-\infty}^{\infty} \zeta(t) u^{3}(r, t) d t-r^{2} \int_{-\infty}^{\infty} \zeta(t) u_{t t}(r, t) d t \\
& =-v+\int_{-\infty}^{\infty} \zeta(t) u^{3}(r, t) d t-r^{2} \int_{-\infty}^{\infty} \zeta_{t t} u(r, t) d t
\end{aligned}
\end{aligned}
$$


If $\zeta$ is as shown in the diagram above, then $\zeta_{t t}$ is either positive or its modulus is less than some constant times $\zeta$. Hence we can arrange that

$$
\int_{-\infty}^{\infty} \zeta(t) u^{3}(r, t) d t-r^{2} \int_{-\infty}^{\infty} \zeta_{t t} u(r, t) d t \leqq \varepsilon v
$$

for $r<\delta(\varepsilon)$. Hence for $r<\delta(\varepsilon)$ we find $r^{2} \frac{d^{2} v}{d r^{2}}<-(1-\varepsilon) v$.

If we substitute $r=e^{\tau}$, this yields

$$
\ddot{v}-\dot{v}<-(1-\varepsilon) v \text {. }
$$

If $u$ does not vanish identically, we may find a $\tau_{0}$ such that $v\left(\tau_{0}\right)>0, \dot{v}\left(\tau_{0}\right)>0$. The following diagram shows the solution $w$ of $\ddot{w}-\dot{w}=-(1-\varepsilon) w$ with the initial condition $w\left(\tau_{0}\right)=v\left(\tau_{0}\right), \dot{w}\left(\tau_{0}\right)=\dot{v}\left(\tau_{0}\right)$.

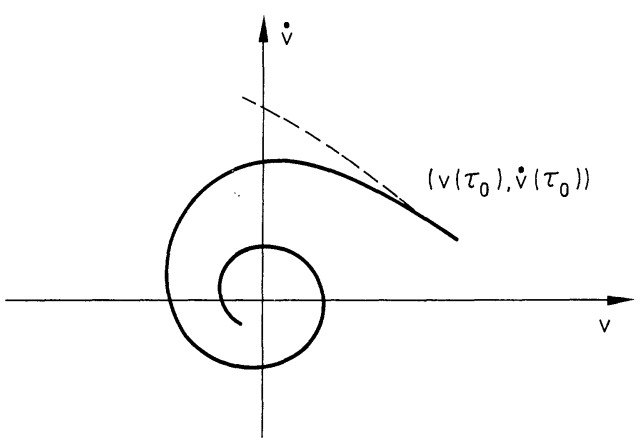

Fig. 1.3

According to (1.1) for $\tau \lesssim \tau_{0}$ the phase plane curve for $v$ is above the curve for $w$ and the two curves do not intersect again as long as $v>0, \dot{v}>0$. This is indicated by the dashed line in the diagram. In particular, we conclude from this that for $\tau<\tau_{0} \dot{v}$ remains strictly positive as long as $v>0$, and hence $v$ must change its sign for a certain finite $\tau_{1}<\tau_{0}$. (It is a priori clear from the definition of $v$ that $\dot{v}$ cannot diverge to $\infty$ for finite $\tau$.) This contradicts the positivity of $v$. Hence $v=0$, i.e. $u=0$ in a whole subdomain of $R_{+}^{2}$. Since $u$ is real analytic, this implies that $u$ vanishes identically.

\section{Solutions which Depend only on $t / r$}

Substituting $t / r=: \sinh \tau$, we obtain the ordinary differential equation

$$
\ddot{u}+\tanh \tau \dot{u}=u^{3}-u
$$

from which we conclude (after multiplication by $\dot{u}$ )

$$
\frac{d}{d \tau}\left(\frac{1}{2} \dot{u}^{2}+\frac{1}{2} u^{2}-\frac{1}{4} u^{4}\right)=-\tanh \tau \dot{u}^{2}
$$


The following diagram shows the lines of constant level for the function $\frac{1}{2} \dot{u}^{2}+\frac{1}{2} u^{2}-\frac{1}{4} u^{4}$.

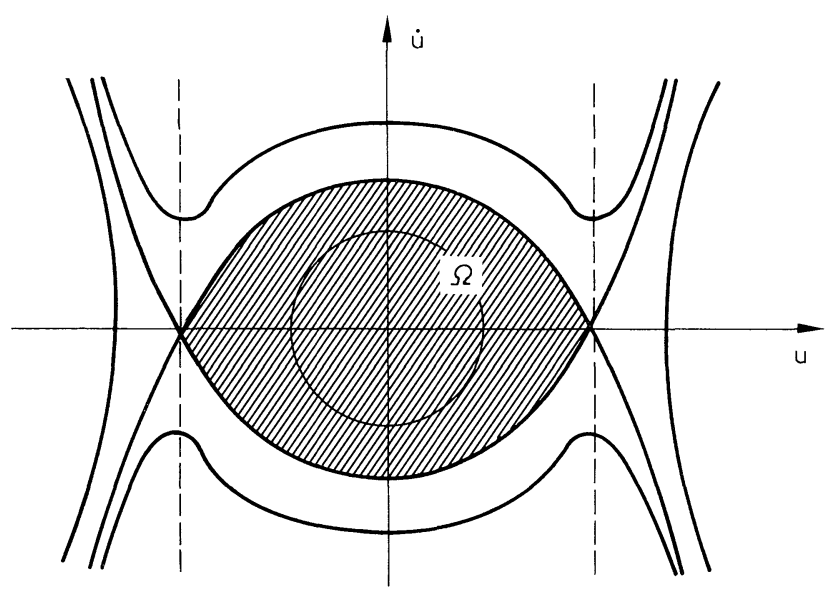

Fig. 2.1

We see immediately that there exists a two dimensional continuum of bounded solutions.

Lemma 2.1. Whenever $(u(0), \dot{u}(0))$ is in $\bar{\Omega} \backslash\{(1,0),(-1,0)\}$, the solution of $(2.1)$ with these initial conditions for $\tau=0$ is bounded and approaches the origin as $\tau \rightarrow \pm \infty$.

We shall next prove

Theorem 2.2. Given any $\lambda$ between -1 and 0 , there exists at least one solution of (2.1) satisfying an initial condition $\dot{u}(0)>0, u(0)=\lambda$ which approaches -1 as $\tau \rightarrow-\infty$ and 0 as $\tau \rightarrow \infty$. For $\lambda=0$ there is a solution approaching 1 as $\tau \rightarrow \infty$ and -1 as $\tau \rightarrow-\infty$, and for each $0<\lambda<1$ there is a solution approaching 1 as $\tau \rightarrow \infty$ and 0 as $\tau \rightarrow-\infty$.

Proof. We confine ourselves to the case $\lambda<0$, the other cases are discussed in the same way.

If $u(0)=\lambda$ and $\dot{u}(0)$ is sufficiently small, then $(u, \dot{u})$ stays in $\Omega$ for every $\tau \in R$.

On the other hand

$$
\begin{aligned}
\frac{d}{d \tau}\left(\frac{1}{2} \dot{u}^{2}+\frac{1}{2} u^{2}-\frac{1}{4} u^{4}\right) & =-\tanh \tau\left(\dot{u}^{2}+u^{2}-\frac{1}{2} u^{4}\right)+\tanh \tau\left(u^{2}-\frac{1}{2} u^{4}\right) \\
& \geqq-\left(\dot{u}^{2}+u^{2}-\frac{1}{2} u^{4}\right) \text { if } \tau>0 \text { and } \quad-1 \leqq u \leqq 1
\end{aligned}
$$

Hence if $u(0)=\lambda$, we find

$$
\dot{u}^{2}(\tau) \geqq e^{-2 \tau}\left(\dot{u}^{2}(0)+\lambda^{2}-\frac{1}{2} \lambda^{4}\right)+\frac{1}{2} u^{4}-u^{2} \geqq e^{-2 \tau}\left(\dot{u}^{2}(0)+\lambda^{2}-\frac{1}{2} \lambda^{4}\right)-\frac{1}{2}
$$

as long as $-1 \leqq u(\tau) \leqq 1$.

We see from this that the solution leaves the strip $-1 \leqq u \leqq 1$ for $\tau>0$, provided $\dot{u}(0)$ is large enough. Similarly, the solution leaves the strip for $\tau<0$, if $\dot{u}(0)$ is large 
enough. A continuity argument now shows that there exists $C>0$, such that the solution $u_{0}$ of $(2.1)$ satisfying $u_{0}(0)=\lambda, \dot{u}_{0}(0)=C$ remains in the strip and approaches 1 as $\tau \rightarrow \infty$ or -1 as $\tau \rightarrow-\infty$. We now derive estimates that make sure that actually $\lim _{\tau \rightarrow-\infty} u_{0}(\tau)=-1$ and $\lim _{\tau \rightarrow \infty} u_{0}(\tau)=0$.

Denoting $u_{0}(-\tau)$ by $v_{0}(\tau)$, we obtain from (2.1)

$$
\begin{gathered}
\left(u_{0}+v_{0}\right) \ddot{+}+\tanh \tau\left(u_{0}+v_{0}\right)=u_{0}^{3}-u_{0}+v_{0}^{3}-v_{0} \\
u_{0}(0)=v_{0}(0)<0, \quad \dot{u}_{0}(0)=-\dot{v}_{0}(0)>0 .
\end{gathered}
$$

For any $\tau>0$ we have $-1<u_{0}, v_{0}<1$, and as long as $\left(u_{0}, \dot{u}_{0}\right),\left(v_{0},-\dot{v}_{0}\right) \notin \Omega$ (which is true for small $\tau$ ) $\dot{u}_{0}>0$ and $\dot{v}_{0}<0$.

The right side of (2.3) is positive for sufficiently small $\tau>0$, i.e.

$$
\frac{d}{d \tau}\left(u_{0}+v_{0}\right)^{\cdot}+\tanh \tau\left(u_{0}+v_{0}\right)^{\circ}>0 \text {. }
$$

Since we have $\left(u_{0}+v_{0}\right)^{*}=0$ [whence $\left(u_{0}+v_{0}\right){ }^{\prime}>0$ ] at $\tau=0$, we may conclude from (2.3) that $\left(u_{0}+v_{0}\right) \geqq 0$ for small enough $\tau$, let us say for $\tau \leqq \tau_{0}$.

As long as $\left(u_{0}, \dot{u}_{0}\right), \quad\left(v_{0},-\dot{v}_{0}\right) \notin \Omega$, the inequality $\left(u_{0}+v_{0}\right)>0$ implies $\dot{u}_{0}>-\dot{v}_{0} \Rightarrow \dot{u}_{0}^{2}>\dot{v}_{0}^{2}$, which, together with (2.2), yields

$$
\begin{aligned}
\frac{1}{2} \dot{u}_{0}^{2}+\frac{1}{2} u_{0}^{2}-\frac{1}{4} u_{0}^{4}<\frac{1}{2} \dot{v}_{0}^{2}+\frac{1}{2} v_{0}^{2}-\frac{1}{4} v_{0}^{4} & \Rightarrow \frac{1}{2} u_{0}^{2}-\frac{1}{4} u_{0}^{4}<\frac{1}{2} v_{0}^{2}-\frac{1}{4} v_{0}^{4} \\
& \Rightarrow\left|u_{0}\right|<\left|v_{0}\right| .
\end{aligned}
$$

This proves that $\left(u_{0}\left(\tau_{0}\right), \dot{u}_{0}\left(\tau_{0}\right)\right) \in \Omega$ or else $\left|u_{0}\left(\tau_{0}\right)\right|<\left|v_{0}\left(\tau_{0}\right)\right|$. If $\tau_{0}=\infty$ or $\left(u_{0}\left(\tau_{0}\right), \dot{u}_{0}\left(\tau_{0}\right)\right) \in \Omega$, this implies the statement of the theorem. So we assume $\tau_{0}<\infty$ and $\left(u_{0}\left(\tau_{0}\right), \dot{u}_{0}\left(\tau_{0}\right)\right) \notin \Omega$. Then $\left|u_{0}\left(\tau_{0}\right)\right|<\left|v_{0}\left(\tau_{0}\right)\right|$ and

$$
u_{0}^{3}\left(\tau_{0}\right)-u_{0}\left(\tau_{0}\right)+v_{0}^{3}\left(\tau_{0}\right)-v_{0}\left(\tau_{0}\right) \leqq 0
$$

This is only possible if $3 v_{0}^{2}-1>0$.

Therefore, $\frac{d}{d \tau}\left(u_{0}^{3}-u_{0}+v_{0}^{3}-v_{0}\right)=\left(3 u_{0}^{2}-1\right) \dot{u}_{0}+\left(3 v_{0}^{2}-1\right) \dot{v}_{0}<0$ as long as $\left|u_{0}\right|<\left|v_{0}\right|$ and $\dot{v}_{0} \leqq-\dot{u}_{0}<0$.

Therefore the right side of (2.3) is negative for $\tau \gtrsim \tau_{0}$, and it remains negative as long as $\left|u_{0}\right|<\left|v_{0}\right|$ and $\dot{v}_{0} \leqq-\dot{u}_{0}<0$. Since $\left(u_{0}+v_{0}\right)^{\circ}=0$ at $\tau=\tau_{0}$, this and (2.3) imply that $\left(u_{0}+v_{0}\right)^{\prime}$ is negative for $\tau>\tau_{0}$, as long as $\dot{v}_{0}$ and $\dot{u}_{0}$ do not change their sign. But this implies that $\dot{v}_{0}$ cannot change its sign unless $\dot{u}_{0}$ does. Therefore either $\dot{u}_{0}$ has a change of sign for some $\tau_{1}>\tau_{0}$, which implies $\left(u_{0}\left(\tau_{1}\right), \dot{u}_{0}\left(\tau_{1}\right)\right) \in \Omega$, or $\dot{u}_{0}+\dot{v}_{0}$ is negative for every $\tau>\tau_{0}$, which implies that $\left|v_{0}\right|-\left|u_{0}\right|$ increases monotonically for $\tau>\tau_{0}$. This concludes the proof.

Remark. In the special case $\lambda=0$, the solution given by the last theorem (which is later proved to be unique) is the well known single meron solution given explicitly by $u=\tanh \tau$ (cf. [4]).

Theorem 2.3. In Theorem 2.2 "at least one" may be replaced by "one and only one".

Proof. (i) If the solutions with the initial conditions $u(0)=\lambda \geqq 0, \dot{u}(0)=a, b$ resp. approach 1 as $\tau \rightarrow \infty$, then all the solutions with an initial condition $u(0)=\lambda$, $a<\dot{u}(0)<b$ have the same behaviour. 
Fig. 2.2

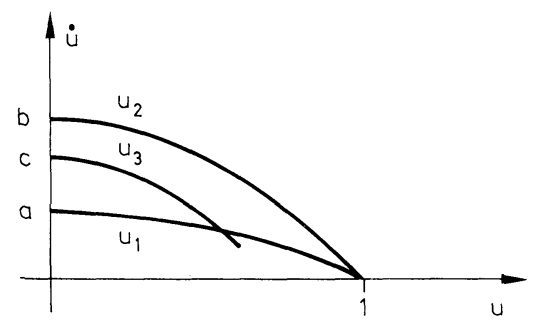

It is obviously sufficient to prove that the solution $u_{3}$ starting from $(\lambda, c)$ never crosses the two other solutions. Assume the contrary as indicated in the diagram, i.e. $\exists \tau_{1}, \tau_{2}>0$ such that $u_{3}\left(\tau_{1}\right)=u_{1}\left(\tau_{2}\right), \dot{u}_{3}\left(\tau_{1}\right)=\dot{u}_{1}\left(\tau_{2}\right)$. Since

$$
\tau_{1}=\int_{\lambda}^{u_{3}\left(\tau_{1}\right)}\left(\dot{u}_{3}\right)^{-1} d u_{3}, \quad \tau_{2}=\int_{\lambda}^{u_{1}\left(\tau_{2}\right)}\left(\dot{u}_{1}\right)^{-1} d u_{1},
$$

we see that $\tau_{1}<\tau_{2}$. Hence (3.1) implies that

$$
\ddot{u}_{3}\left(\tau_{1}\right)=-\tanh \tau_{1} \cdot \dot{u}_{3}\left(\tau_{1}\right)+u_{3}^{3}\left(\tau_{1}\right)-u_{3}\left(\tau_{1}\right)>\ddot{u}_{1}\left(\tau_{2}\right),
$$

which is an apparent contradiction. The same argument shows that $u_{3}$ and $u_{2}$ cannot cross.

(ii) Given $T>0$ large enough, there exists a neighbourhood $U$ of (1.0) and an analytic curve $C$ in $U$ passing through the point $(1,0)$, such that for $(u(T), \dot{u}(T))$ in $U$ the solution $u$ of (2.1) with this initial condition approaches 1 as $\tau \rightarrow \infty$ iff $(u(T), \dot{u}(T)) \in C$.

From (ii) the theorem follows easily, since the mapping $(u(T), \dot{u}(T)) \rightarrow(u(0), \dot{u}(0))$ is (locally) analytic and hence takes $C$ to an analytic curve $\tilde{C}$. But an analytic curve cannot contain an interval on the line $u=\lambda$.

So it remains to prove (ii). After the substitution $u-1=v,(2.1)$ reads

$$
\ddot{v}+\dot{v}-2 v=v^{3}+3 v^{2}+(1-\tanh \tau) \dot{v} .
$$

We rewrite this equation as an operator equation

$$
L v=M(v) \quad L, M: C_{b}^{2}([T, \infty)) \rightarrow C_{b}([T, \infty)) .
$$

$L$ has a one dimensional nullspace and full range, i.e. there exists a right inverse $\hat{L}$ and we may rewrite $(2.5)$ in the form

$$
v=\hat{L} M(v)+f,
$$

where $f$ is in the nullspace of $L$. If $T$ is large enough, we conclude from the implicit function theorem that locally (2.6) has a unique analytic resolution $v=v(f)$. Since the projection $v \rightarrow(v(T), \dot{v}(T))$ is a continuous linear operator from $C_{b}^{2}([T, \infty))$ into $R^{2}$, this implies (ii).

\section{Solutions which are Periodic in $t$}

Substituting $r=e^{x}$ and denoting differerentiation w.r.t. $x$ by ', we obtain from (0.1)

$$
u^{\prime \prime}-u^{\prime}+e^{2 x} u_{t t}+u=u^{3} \text {. }
$$


Using the Fourier expansion $u=\sum_{k \in Z} u_{k}(x) e^{i k \omega t}$ we find

$$
u_{k}^{\prime \prime}-u_{k}^{\prime}-k^{2} \omega^{2} e^{2 x} u_{k}+u_{k}=\left(u^{3}\right)_{k}=\sum_{l, m \in Z} u_{k-l}(x) u_{l-m}(x) u_{m}(x)
$$

We are looking for small solutions of (3.2) in the space

$$
l^{1}\left(C_{b}(R)\right):=\left\{u \mid u_{k} \text { continuous, } \sum_{k \in Z} \sup _{x \in R}\left|u_{k}(x)\right|<\infty\right\} .
$$

We see that (3.2) is of the form $Q u=u^{3}$, and we shall prove that there exists an operator $M \in \mathscr{L}\left(l^{1}\left(C_{b}(R)\right)\right.$ such that $Q M=1$. ( $M$ has regularizing properties so that $Q$ is defined on the range of $M$.) The equation

$$
u-M u^{3}=f
$$

has a unique solution $u=u(f)$ in a neighbourhood of 0 , and this solution gives a solution of (3.2) iff $f$ is in the kernel of $Q$. Hence we obtain a one-to-one correspondence between small solutions of (3.2) and members of the kernel $N$ of the linearization $Q$. We now construct $M$. Consider the problem

$$
u_{k}^{\prime \prime}-u_{k}^{\prime}+u_{k}-k^{2} \omega^{2} e^{2 x} u_{k}=v_{k}(x) \quad\left(v_{k}\right) \in l^{1}\left(C_{b}(R)\right) .
$$

For $k=0$ this has a unique solution $u_{0}=M_{0} v_{0} \in C_{b}(R)$. So let now be $k \neq 0$. Substituting $\zeta=x-x_{k}^{\prime}$, where $x_{k}^{\prime}$ is defined by $\exp \left(2 x_{k}^{\prime}\right)=(k \omega)^{-2}$, we reduce the problem to the equation

$$
u^{\prime \prime}-u^{\prime}+u-e^{2 \zeta} u=v
$$

So we have to construct a linear operator $\tilde{M}$ in $\mathscr{L}\left(C_{b}(R)\right)$, such that $u=\tilde{M} v$ solves (3.4).

With $r=e^{\zeta}(3.4)$ reads

$$
u_{r r}-u+r^{-2} u=r^{-2} v .
$$

If $r_{0}$ is sufficiently large, the term $r^{-2} u$ can be treated as a perturbation, and from the characteristic exponents of $u_{r r}-u=0$ we see that (3.5) has a unique bounded solution for $r \geqq r_{0}$ which obeys the initial condition $u_{r}\left(r_{0}\right)=0$. This solution depends continuously on $v$, i.e. there exists a constant $C$ such that

$$
\sup _{r \geqq r_{0}}|\mathrm{u}(\mathrm{r})| \leqq C \sup _{r \geqq r_{0}}|v(r)| \text {. }
$$

Now continue this solution to the left side according to Eq. (3.4). This gives a solution $u$ in any interval $-\zeta_{0} \leqq \zeta<\infty$, which depends continuously on $v$ :

$$
\sup _{\zeta \geqq-\zeta_{0}}|u(\zeta)| \leqq C\left(\zeta_{0}\right) \sup _{\zeta \geqq \zeta_{0}}|v(\zeta)|
$$

If we choose $\zeta_{0}$ large enough, $e^{2 \zeta}$ becomes arbitrarily small for $\zeta<-\zeta_{0}$, so that now the term $e^{2 \zeta} u$ can be treated as a perturbation, and from the characteristic exponents of $u^{\prime \prime}-u^{\prime}+u=0$ we see that on $\left(-\infty,-\zeta_{0}\right)$ the Eq. (3.4) defines $u$ as a continuous function of $v, u\left(-\zeta_{0}\right)$ and $u^{\prime}\left(-\zeta_{0}\right)$. So taking everything together we have found a solution $u$ of (3.4) depending continuously on $v$ :

$$
\sup _{\zeta \in R}|u(\zeta)| \leqq C^{\prime} \sup _{\zeta \in R}|v(\zeta)| .
$$


The solution $u$ constructed in this manner is defined as $\tilde{M} v$, which completes the construction of $M$. The elements of $N$ are obtained by the same construction as above, if we take $v=0$ and replace the condition $u_{r}\left(r_{0}\right)=0$ by $u_{r}\left(r_{0}\right)=c, c \neq 0$. This shows that for every $k \neq 0$ we find a one-dimensional nullspace. We thus have an infinite dimensional nullspace and hence an infinite dimensional manifold of nontrivial solutions of $(3.1)$.

The condition $\lim _{x \rightarrow-\infty} u_{k}(x)=0, k \in Z$ defines a closed subspace $Y$ of $l^{1}\left(C_{b}(R)\right)$, which is mapped into itself by $M$. Since the members of the kernel of $Q$ belong to $Y$, we see from (3.3) that our solutions are in $Y$ as well.

We have thus proved

Theorem 3.1. For any given period There exists an infinite dimensional manifold of solutions to $(0.1)$ which are T-periodic w.r.t. $t$ and obey the boundary condition $\lim u(r, t)=0$ uniformly in $t$.

\section{Nonperiodic Solutions}

In the argument above we replace Fourier expansion by the Fourier integral, i.e.

$$
u(x, k)=\int_{-\infty}^{\infty} u(x, t) e^{-i k t} d t
$$

and we replace the space $l^{1}\left(C_{b}(R)\right)$ by

$$
\begin{aligned}
L^{1}\left(C_{b}(R)\right)= & \left\{u ( x , k ) \left\{u \text { measurable, } u(\cdot, k) \in C_{b}(R)\right.\right. \text { for almost every } \\
& \left.k, \int_{x} \sup _{x}|u(x, k)| d k<\infty\right\} .
\end{aligned}
$$

The operator $M$ is constructed exactly as above, however, we have to discuss some technical details. First we make sure that the definition of $L^{1}\left(C_{b}(R)\right)$ makes sense:

Lemma 4.1. If $u$ is measurable and $u(\cdot, k) \in C_{b}(R)$ for almost every $k$, then $\sup u(x, k)$ is measurable.

Proof. $\left\{k \mid \sup _{x} u(x, k)>K\right\}=\bigcup_{x \in Q}\{k \mid u(x, k)>K\}$ modulo a null set.

Lemma 4.2. $L^{1}\left(C_{b}(R)\right.$ ) (with natural norm) is a Banach space.

Proof. Let $\left\{u_{m}\right\}_{m \in N}$ be a Cauchy sequence in $L^{1}\left(C_{b}(R)\right)$. Since after passing to a subsequence $L^{1}$-convergence implies convergence a.e.,

$$
\lim _{m, n \rightarrow \infty} \sup _{x}\left|u_{n}(x, k)-u_{m}(x, k)\right|=0
$$

for almost every $k$; hence $u_{m}$ converges to a function $u$ uniformly in $x$ for a.e. $k$. Since $u=\lim _{m \rightarrow \infty} u_{m}$ except for a null set, $u$ is measurable, and clearly $u(\cdot, k) \in C_{b}(R)$ for a.e. $k$. It remains to be shown that

$$
\int \sup _{x}\left|u(x, k)-u_{m}(x, k)\right| d k \rightarrow 0 \quad \text { as } \quad m \rightarrow \infty
$$


$v_{n}(k)=\sup _{x}\left|u_{n}(x, k)-u_{m}(x, k)\right|$ is a Cauchy sequence in $L^{1}$ and hence convergent to $\sup \left|u(x, k)-u_{m}(x, k)\right|$. Therefore we find

$$
\int \sup _{x}\left|u(x, k)-u_{m}(x, k)\right| d k \leqq \limsup _{n \rightarrow \infty} \int \sup _{x}\left|u_{n}(x, k)-u_{m}(x, k)\right| d k
$$

which implies the lemma.

Lemma 4.3. $M$ (constructed as before) maps $L^{1}\left(C_{b}(R)\right)$ into itself.

Proof. Clearly the only difficult step is to show that $M u$ is measurable. Since the transformation $(x, k) \rightarrow\left(\zeta+x_{k}^{\prime}, k\right)$ transforms measurable functions to measurable functions, it is sufficient to prove that $\tilde{M} u$ is measurable. But $L^{1}\left(C_{b}(R)\right) \subset C_{b}\left(L^{1}(R)\right)$ [the space of all bounded continuous functions $R \rightarrow L^{1}(R)$ ], and one easily concludes from the construction of $\tilde{M}$ that $\tilde{M}$ maps $C_{b}\left(L^{1}(R)\right)$ into itself. [To see this, we only have to reinterpret $u$ and $v$ in (4.4) as elements of $L^{1}(R)$.] Since all elements of $C_{b}\left(L^{1}(R)\right)$ are measurable functions, the lemma is proved.

From Lemmas 4.1 to 4.3 we conclude that we may now perform the same construction as in Sect. 3 and obtain an infinite dimensional manifold of nonperiodic solutions.

The condition $\lim _{x \rightarrow-\infty} \int \sup _{\zeta \leqq x}|u(\zeta, k)| d k=0$ defines a closed subspace of $L^{1}\left(C_{b}(R)\right)$, which is mapped into itself by $M$, and as before we conclude from this fact that the solutions we have constructed vanish in the limit $x \rightarrow-\infty$.

Altogether we have proved:

Theorem 4.4. There exists an infinite dimensional manifold of solutions to (0.1), which are nonperiodic in $t$ and obey the boundary condition $\lim _{r \rightarrow 0} u(r, t)=0$ uniformly with respect to $t$.

\section{Some Remarks on the Physical Significance of our Solutions}

Equation (0.1) has been derived [1] from the SU(2) Yang-Mills equations by the special ansatz $A=d \theta, \phi=\Theta(0, u)$, where $\Theta$ is the matrix

$$
\Theta=\left(\begin{array}{rr}
\cos \theta & \sin \theta \\
-\sin \theta & \cos \theta
\end{array}\right) .
$$

If $\theta$ has no singularities, this leads to a vanishing charge density. Therefore, all the solutions having continuous boundary values (particularly $u=0$ ) on the $t$-axis may be interpreted as solutions of the Yang-Mills equations with zero charge. The physically more interesting solutions are those assuming the boundary values \pm 1 on the $t$-axis. The solutions considered in [1] are of this type, and there the singularities on the $t$-axis are compensated by singularities of $\theta$ in such a way that $\phi$ is constant on the $t$-axis. These singularities in $\theta$ lead to point charges [1] located on the $t$-axis. We have seen in Sect. 2 that a special solution with boundary values \pm 1 can be obtained as a limiting case of solutions with boundary value 0 . We suspect that in a similar way solutions with boundary values \pm 1 can be found 
on the boundary of the manifolds, the existence of which we have established in Sects. 3 and 4.

Acknowledgements. I thank Professor K. Kirchgässner, who encouraged me to write this paper, and I thank my colleague W. Häffner for a valuable discussion on the physical aspects of the problem. Finally, I thank Professor H. Haken, in whose institute I did this work, and I thank the Volkswagen Foundation for financial support within the project of Synergetics.

\section{References}

1. Glımm, J., Jaffe, A.: Multiple meron solutions of the classical Yang-Mills equation. Phys. Lett. 73B, 167-170 (1978)

2. Jonsson T. et al.: An existence theorem for multimeron solutions to classical Yang-Mills field equations. Commun. Math. Phys. 68, 259-273 (1979)

3. Dinghas, A.: Vorlesungen uber Funktionentheorıe. Berlin, Göttingen, Heidelberg: Springer 1961

4. Alfaro, V. de, Fubini, S., Furlan, G. : Phys. Lett. 65B, 163 (1976)

5. Protogenov, A.P.: Bag and multimeron solutions of the classical Yang-Mills equations. Phys. Lett 87B, 80-82 (1979)

6. Protter, M., Weinberger, H.: Maximum principles in differential equations. Englewood Cliffs: Prentice Hall 1967

Communicated by A. Jaffe

Received January 17, 1980 
